Check for updates

Cite this: RSC Adv., 2019, 9, 16058

\title{
Optimized synthesis of novel hydrogel for the adsorption of copper and cobalt ions in wastewater
}

\begin{abstract}
Wei Zhang, Lishuang Hu, (D) * Shuangqi Hu and Yang Liu
Metal ions in wastewater endanger the environment and even human life. In this study, an optimized method was used to synthesize an excellent hydrogel to treat these metal ions. The samples were characterized by Fourier transform infrared spectroscopy (FTIR), scanning electron microscopy (SEM) and thermogravimetric analysis (TGA), and applied to treat the $\mathrm{Cu}(॥)$ and $\mathrm{Co}(॥)$ ions in wastewater. In the adsorption experiment, the influential factors such as $\mathrm{pH}$, adsorption time, adsorbent dosage and concentration of heavy metal ions and regeneration efficiency were evaluated, and the adsorption kinetics, isotherms and thermodynamics were studied. The orthogonal optimization results show that the best condition for synthesis was when the degree of neutralization of acrylic acid $(A)$ was $70 \%$, the quantity of glucose $(B)$ was $0.2 \mathrm{~g}$, the quantity of chitosan $(C)$ was $0.05 \mathrm{~g}$, and the quantity of initiator $(D)$ was $0.03 \mathrm{~g}$. The influence of the four factors was in the order $D>B>C>A$. The adsorption performance was optimal under neutral conditions and the dosage of $0.02 \mathrm{~g}$ adsorbent was chosen as the best. Experiments show that the composite hydrogels exhibited excellent performance under optimal conditions: at $20{ }^{\circ} \mathrm{C}$ and $\mathrm{pH}=7$, the adsorption capacity of $100 \mathrm{mg} \mathrm{L}^{-1}$ of $\mathrm{Cu}(\mathrm{II})$ by $0.01 \mathrm{~g}$ hydrogel was $286 \mathrm{mg} \mathrm{g}^{-1}$. The adsorption process of heavy metal ions by hydrogels conforms to pseudo-second-order kinetics and Langmuir isotherm model, which indicate a spontaneous endothermic reaction. Moreover, after five cycles, the removal rates of $\mathrm{Cu}\left({ }^{\prime \prime}\right)$ and $\mathrm{Co}(I)$ were $81 \%$ and

$74.8 \%$, respectively.
\end{abstract}

Received 10th January 2019 Accepted 27th April 2019

DOI: $10.1039 / c 9 r a 00227 h$

rsc.li/rsc-advances

\section{Introduction}

In recent years, with the rapid development of industries, the use of metal minerals has become more and more frequent. ${ }^{1-5} \mathrm{~A}$ large number of harmful substances are constantly entering the water bodies due to human activities, and water pollution is becoming increasingly serious. ${ }^{6-13}$ Among them, due to the extensive toxic effects of heavy metal pollutants, water pollution has caused widespread concern. Therefore, the removal of heavy metal ions has become one of the hot topics in the scientific community. ${ }^{14,15}$

At present, the treatment of heavy metal ions in wastewater is being studied by many scholars in various countries. According to different research areas and mechanisms of the treatment measures, the methods can be roughly divided into chemical treatment, physical treatment and biological treatment. ${ }^{16-18}$ At present, the most effective environmental treatment method is the adsorption method. Adsorptive materials are generally divided into inorganic, organic and composite materials, of which composite materials are the most widely used. Compared with inorganic materials and organic materials, the composite

School of Environmental and Safety Engineering, North University of China, 030051 Taiyuan, China. E-mail: hulish766@foxmail.com; Fax: +86 03513920504; Tel: +86 03513920504 materials have better adsorption performance that is stable. A new type of composite material, hydrogel, can constitute many organic materials, and its super absorbency bestows it with a great potential in water treatment. ${ }^{1920}$ Badawi $^{18}$ et al. synthesized a novel hydrogel by blending tannic acid with chitosan and studied the adsorption of $\mathrm{Al}(\mathrm{III})$ and $\mathrm{Pb}$ (II). The results were very significant. Karthik ${ }^{21}$ et al. used polyaniline grafted in chitosan to prepare a hydrogel and studied the adsorption of $\mathrm{Pb}(\mathrm{II})$ and $\mathrm{Cd}(\mathrm{II})$. Misra ${ }^{22}$ et al. used iminodiacetic acid functionalization to develop a hydrogel from a copolymer of styrene and divinylbenzene, and investigated its adsorption capacity towards $\mathrm{Ni}(\mathrm{II}), \mathrm{Pb}$ (II) and $\mathrm{Cd}(\mathrm{II}) . \mathrm{Zhou}^{23}$ et al. studied the crosslinking of starch and polyacrylic acid in detail, and then, modified it by using triethylenetetramine to obtain a new amino starch/polyacrylic acid adsorbent. The adsorption capacity towards cadmium ions was found to be as high as $256.4 \mathrm{mg} \mathrm{g}^{-1}$. $\mathrm{Zhou}^{24}$ et al. synthesized a monolithic super macroporous hydrogel by thermally inducing high internal phase emulsion polymerization to graft acrylic acid onto the chitosan backbone. The hydrogel was found to have higher adsorption rates for $\mathrm{Cu}$ (II) and $\mathrm{Pb}$ (II) with high adsorption capacities of $302.01 \mathrm{mg}$ $\mathrm{g}^{-1}$ and $611.94 \mathrm{mg} \mathrm{g}^{-1}$, respectively, and could be recycled five times. Karawi ${ }^{25}$ et al. used potassium persulfate as an initiator to copolymerize acrylamide by grafting it onto chitosan to synthesize a chitosan-polyacrylamide complex. The factors 
influencing copolymerization such as initiator concentration, acrylamide monomer to chitosan ratio and reaction temperature were optimized, and the adsorption performance of chitosan-polyacrylamide complex towards $\mathrm{Cu}(\mathrm{II})$ was investigated. ${ }^{26}$

Being a long-chain polymer compound, chitosan contains a large number of functional groups such as hydroxyl groups, ethylamino groups, electron-rich pyran rings and bridge oxygens. Therefore, chitosan has a large number of hydrogen bonds in the molecule and between molecules, ${ }^{27-35}$ thereby forming a network-like structure with stable chelation of metal ions. These properties make it suitable to be often used with monomers to synthesize hydrogels. ${ }^{36,37}$ The most widely distributed monosaccharide in nature, glucose, has the advantages of abundant resources, low price and good biodegradability. Its molecular structure contains a large number of hydroxyl groups that imparts strong hydrophilicity. These characteristics make it a potential raw material for the preparation of gel adsorption materials. ${ }^{38-44}$ In this study, chitosan, glucose, and acrylic acid were used as monomers, potassium persulfate was used as the initiator, and $N^{\prime} N$-methylenebisacrylamide was used as the crosslinking agent. An ultrasonication-assisted method was used to prepare the new hydrogels and was optimized by the orthogonal method. The samples were characterized by Fourier transform infrared spectroscopy (FTIR), scanning electron microscopy (SEM) and thermogravimetric analysis (TGA). The adsorption of $\mathrm{Cu}(\mathrm{II})$ and $\mathrm{Co}(\mathrm{II})$ ions in wastewater was carried out using the prepared new hydrogel. In the adsorption experiment, factors such as the $\mathrm{pH}$ value, adsorption time, adsorbent dosage, heavy metal ion concentration and recycling capacity were investigated, and the adsorption kinetics and thermodynamic properties were studied.

\section{Experimental}

\subsection{Materials}

Acrylic acid (Sinopharm Chemical Reagent Co., Ltd.), chitosan (Sinopharm Chemical Reagent Co., Ltd.) and glucose (Sinopharm Chemical Reagent Co., Ltd.) were used as the polymer monomers. Potassium persulfate (Sinopharm Chemical Reagent Co., Ltd.) was used as the initiator. $N N^{\prime}$-Methylenebisacrylamide (Sinopharm Chemical Reagent Co., Ltd, Shanghai, China) was the cross-linking agent. Other reagents such as sodium hydroxide, ethanol, copper nitrate pentahydrate and cobalt nitrate tetrahydrate were purchased from Shanghai McLean Biochemical Co., Ltd.

\subsection{Optimization of hydrogel synthesis}

Of the many factors affecting the properties of hydrogels during their synthesis, the following four factors are the most important: the neutralization degree of acrylic acid $(A)$, the quantity of glucose used in polymerization $(B)$, the quantity of chitosan used in polymerization $(C)$ and the quantity of initiator $(D)$. There are many drawbacks in optimizing these factors using the traditional single-factor variable method. ${ }^{45}$ The numerous experiments and tedious operation will increase the errors in the experimental results. Orthogonal optimization can balance sampling within the range of factors so that each experiment has a strong representative. Because each orthogonal experiment is the best choice point, which guarantees the accuracy of the experimental results and greatly reduces the number of experiments, these experiments can often better achieve the experimental purpose.

Sodium hydroxide was dissolved in water and neutralized in an ice bath with $5 \mathrm{~mL}$ acrylic acid. After neutralization, chitosan, glucose and potassium persulfate were added and dissolved by stirring with a glass rod. After dissolution, the mixture was heated to $70^{\circ} \mathrm{C}$ for $3 \mathrm{~h}$ in an ultrasound apparatus. Then, the unreacted substances were soaked and cleaned with anhydrous ethanol. Finally, the hydrogels were obtained by vacuum drying. The quantity of the substances involved are listed in the following orthogonal tables (Table 1).

\subsection{Experimental synthesis mechanism}

The active sites of chitosan and glucose $\left(-\mathrm{OH},-\mathrm{COOH},-\mathrm{NH}_{2}\right)$ are prone to cleavage under the action of heat and initiator. First, the initiator was dissolved in water to produce sulfate radicals, which promote the production of $\mathrm{OH}^{*}$ from water molecules. $\mathrm{OH}^{\cdot}$ attacks the $-\mathrm{OH},-\mathrm{COOH},-\mathrm{NH}_{2}$ and $-\mathrm{CH}_{3}$ groups on chitosan and glucose, which in turn dehydrogenates and creates new free radical sites. Acrylic acid further attacks these sites one after the other to produce polymeric chains, and finally, they are joined together by the action of a cross-linker to form a polymer. The unreacted monomer was washed away with distilled water to obtain the hydrogel (Fig. 1).

\subsection{Characterization}

For the determination of functional groups in the samples, FTIR spectra of the samples were recorded on a FTIR-850 spectrometer from Tianjin Gangdong Company. The wavenumbers ranged from 4000 to $400 \mathrm{~cm}^{-1}$. The morphology of the samples was analyzed using JSM-7800F, Japan Electronics Corporation. The samples were analyzed by thermogravimetric analysis (TGA) with an STA 449C integrated thermal analyzer. The temperature was varied from room temperature to $800{ }^{\circ} \mathrm{C}$.

Table 1 The 4-element 3-level orthogonal experiment design

\begin{tabular}{lllll}
\hline Factors & $A(\%)$ & $B(\mathrm{~g})$ & $C(\mathrm{~g})$ & $D(\mathrm{~g})$ \\
\hline 1 & 60 & 0.1 & 0.05 & 0.01 \\
2 & 60 & 0.2 & 0.1 & 0.03 \\
3 & 60 & 0.3 & 0.15 & 0.05 \\
4 & 70 & 0.1 & 0.1 & 0.05 \\
5 & 70 & 0.2 & 0.15 & 0.01 \\
6 & 70 & 0.3 & 0.05 & 0.03 \\
7 & 80 & 0.1 & 0.15 & 0.03 \\
8 & 80 & 0.2 & 0.1 & 0.05 \\
9 & 80 & 0.3 & 0.05 & 0.01
\end{tabular}




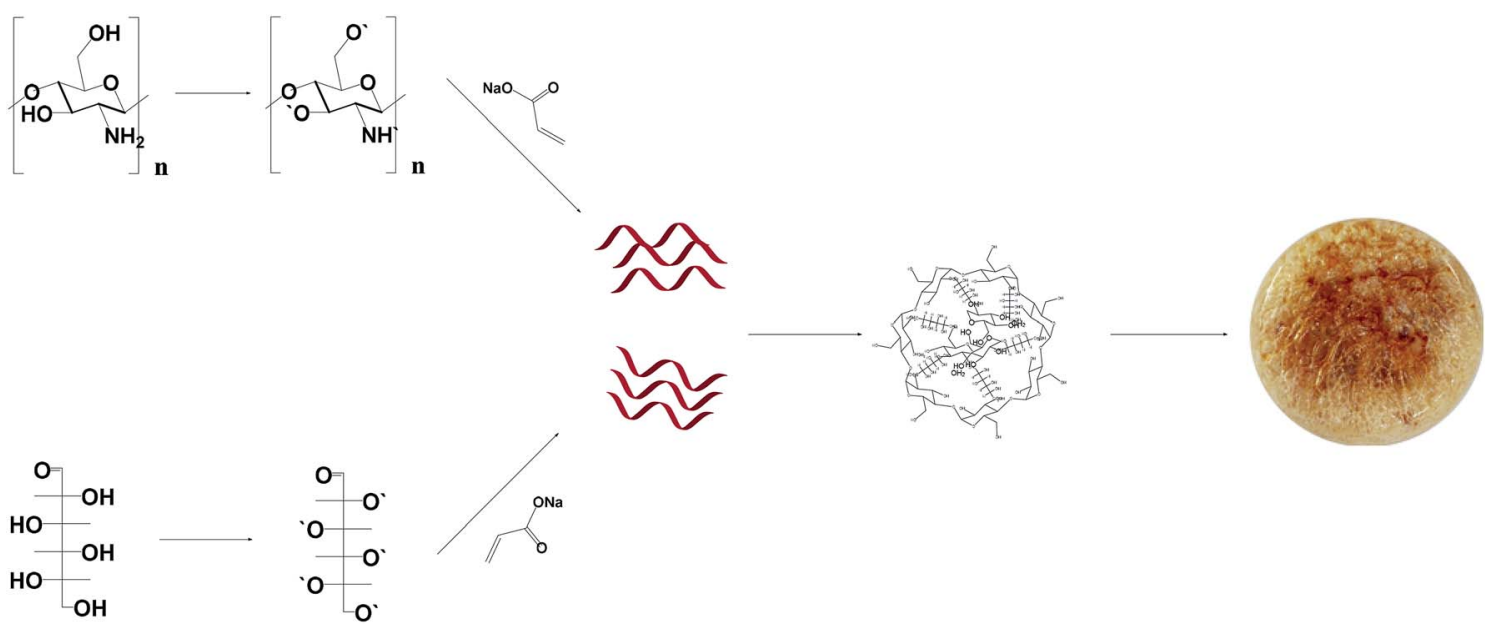

Fig. 1 Synthesis mechanism of hydrogels.

\subsection{Adsorption experiment}

The adsorption performance of the hydrogels, in this work, was studied from five aspects: temperature, dosage of hydrogel, adsorption time, concentration of heavy metal ions and $\mathrm{pH}$. At a certain temperature $\left(20-60{ }^{\circ} \mathrm{C}\right), 50 \mathrm{~mL}$ solution of $100 \mathrm{mg} \mathrm{L}{ }^{-1}$ heavy metal ion ( $\mathrm{Co}(\mathrm{II}), \mathrm{Cu}(\mathrm{II})$ ) concentration was evaluated by adding $0.01 \mathrm{~g}$ hydrogel and allowing to reach the adsorption equilibrium. To $50 \mathrm{~mL}$ of $100 \mathrm{mg} \mathrm{L}^{-1}$ heavy metal ion (Co(II), $\mathrm{Cu}(\mathrm{II}))$ solution, a certain amount (0.01-0.06 g) of hydrogel was added. After reaching the adsorption equilibrium, the concentration of heavy metal ions was measured. The $0.01 \mathrm{~g}$ hydrogel sample was weighed and added into $50 \mathrm{~mL}$ of the $100 \mathrm{mg} \mathrm{L}^{-1}$ solution of heavy metal ions (Co(II), Cu(II)), and the concentrations of heavy metal ions were measured at $5 \mathrm{~min}, 20 \mathrm{~min}$, $30 \mathrm{~min}, 60 \mathrm{~min}, 90 \mathrm{~min}, 120 \mathrm{~min}, 150 \mathrm{~min}$ and $180 \mathrm{~min}$. To $50 \mathrm{~mL}$ of heavy metal ion solution with a certain concentration (100 mg L ${ }^{-1}, 300 \mathrm{mg} \mathrm{L}^{-1}, 500 \mathrm{mg} \mathrm{L}^{-1}, 700 \mathrm{mg} \mathrm{L}^{-1}, 900 \mathrm{mg} \mathrm{L}^{-1}$, or $1100 \mathrm{mg} \mathrm{L}^{-1}$ ), $0.01 \mathrm{~g}$ hydrogel was added, and then, the concentration of heavy metal ions after adsorption was measured. $0.01 \mathrm{~g}$ hydrogel sample was weighed and added into $50 \mathrm{~mL}$ of the $100 \mathrm{mg} \mathrm{L}^{-1}$ heavy metal ion (Co(II), Cu(II)) solution at a certain $\mathrm{pH}(1-7)$, and then, the concentration of heavy metal ions after adsorption was measured. The concentration of heavy metal ions was measured by a TAS-986 atomic absorption spectrophotometer. The following formula was used to calculate the adsorption capacity $q_{\mathrm{e}}$ of the hydrogels.

$$
q_{\mathrm{e}}=\frac{\left(C_{0}-C_{\mathrm{e}}\right) V}{m}
$$

\section{Results and discussion}

\subsection{Optimization of parameters}

The optimal conditions from the orthogonal optimization table is $A(70), B(0.2), C(0.05)$, and $D(0.03)$ (Table 2). The influence of the four factors was in the order $D>B>C>A$. It can be seen from the results that the role of initiator was the most important. The initiation of chain reaction by the initiator had a great impact on the successful grafting of chitosan and glucose onto the hydrogel. Secondly, the influence of glucose, i.e., the hydroxyl groups on glucose had a great influence on the chelation of heavy metal ions, which affected the ability of the hydrogel to adsorb heavy metal ions. It can also be seen from the table that the addition of chitosan and the neutralization degree of acrylic acid also had some influence on the hydrogel adsorption performance. The hydrogel was synthesized according to the orthogonal experimental results. At $20{ }^{\circ} \mathrm{C}$ and $\mathrm{pH}=7$, the adsorption of $100 \mathrm{mg} \mathrm{L}^{-1} \mathrm{Cu}(\mathrm{II})$ solution with $0.01 \mathrm{~g}$ hydrogel was $286 \mathrm{mg} \mathrm{g}^{-1}$.

\subsection{FTIR}

The functional groups of each substance were determined by FTIR spectroscopy to determine whether the hydrogels were successfully grafted with glucose and chitosan. It can be seen from spectrum (a) of Fig. 2 that $-\mathrm{OH},-\mathrm{NH}_{2}$ and $\mathrm{C}-\mathrm{O}-\mathrm{C}$, which are the functional groups on chitosan, appeared in the infrared spectrum. Corresponding absorption peaks also appeared at the respective positions in spectrum (c) of Fig. 2, indicating that chitosan had been grafted onto the hydrogel. Glucose is a polyhydroxy compound having a -CHO group, and a corresponding absorption peak appeared in spectrum (b) shown in Fig. 2. In

Table 2 Result of the optimization experiment

\begin{tabular}{llllll}
\hline Factors & $A(\%)$ & $B(\mathrm{~g})$ & $C(\mathrm{~g})$ & $D(\mathrm{~g})$ & Result $\left(\mathrm{q}_{\mathrm{e}} / \mathrm{mg} \mathrm{g}^{-1}\right)$ \\
\hline 1 & 60 & 0.1 & 0.05 & 0.01 & 220 \\
2 & 60 & 0.2 & 0.1 & 0.03 & 240 \\
3 & 60 & 0.3 & 0.15 & 0.05 & 193 \\
4 & 70 & 0.1 & 0.1 & 0.05 & 189 \\
5 & 70 & 0.2 & 0.15 & 0.01 & 250 \\
6 & 70 & 0.3 & 0.05 & 0.03 & 269 \\
7 & 80 & 0.1 & 0.15 & 0.03 & 200 \\
8 & 80 & 0.2 & 0.1 & 0.05 & 215 \\
9 & 80 & 0.3 & 0.05 & 0.01 & 234 \\
$K_{1}$ & 217 & 203 & 234 & 234 & \\
$K_{2}$ & 236 & 235 & 221 & 236 & \\
$K_{3}$ & 216 & 232 & 214 & 199 & \\
Range & 19 & 32 & 20 & 37 &
\end{tabular}



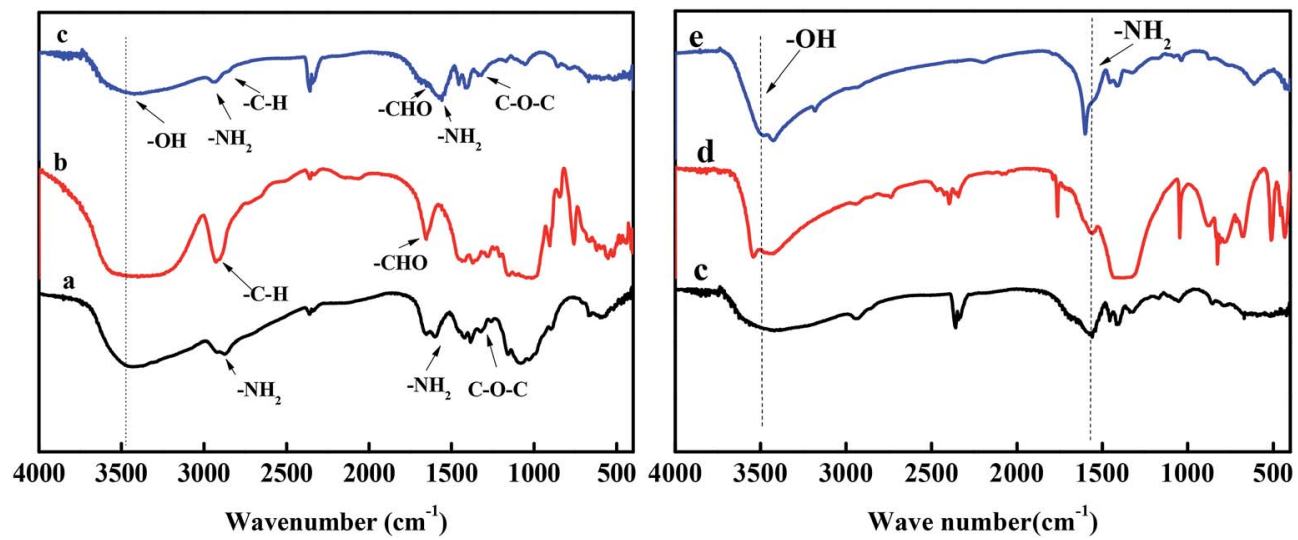

Fig. 2 FTIR spectra of (a) chitosan, (b) glucose, (c) hydrogel, (d) Cu-hydrogel and (e) Co-hydrogel.

the infrared spectrum of the hydrogel, the absorption peak corresponding to the-CHO group was found, which proved that glucose was successfully grafted onto the hydrogel. The hydrogel was applied to remove $\mathrm{Cu}(\mathrm{II})$ and $\mathrm{Co}(\mathrm{II})$ in wastewater. Comparing (c), (d), and (e) spectra in Fig. 2, it was found that the absorption peaks corresponding to - $\mathrm{OH}$ in $\mathrm{Cu}$-hydrogel and Cohydrogel had shifted. The absorption peaks corresponding to $-\mathrm{NH}_{2}$ in Cu-hydrogel and Co-hydrogel were shifted or merged with other absorption peaks. The direct cause of these changes in the absorption peak positions is the sequestration of heavy metal ions with the $\mathrm{O}$ and $\mathrm{N}$ atoms in these functional groups.

\subsection{SEM}

Hydrogel is a kind of polymer network system, which is soft in nature, can keep a certain shape and absorb a large amount of water. ${ }^{44}$ In our experiment, the hydrogel was placed in a vacuum drying chamber to dry and dehydrate. The water molecules evaporated from the inside of the hydrogel and a mesoporous structure was formed on the surface of the hydrogel. Due to the difference in water content at different positions of the hydrogel, the difference in water loss during the drying process leads to wrinkles on the surface of hydrogels. ${ }^{46,47}$

From Fig. 3A and B, it is clear that there were many holes of different sizes and folds in some positions on the surface of the hydrogel. These structures of the hydrogel provide more active sites for adsorption, which is one of the reasons why hydrogels are superior to other inorganic materials as adsorbents.

In Fig. $3 \mathrm{C}$ and $\mathrm{D}$, it is seen that there were no holes on the surface of the hydrogel. A thin film appeared on the surface of the hydrogels. After analysis, these thin films were found to be

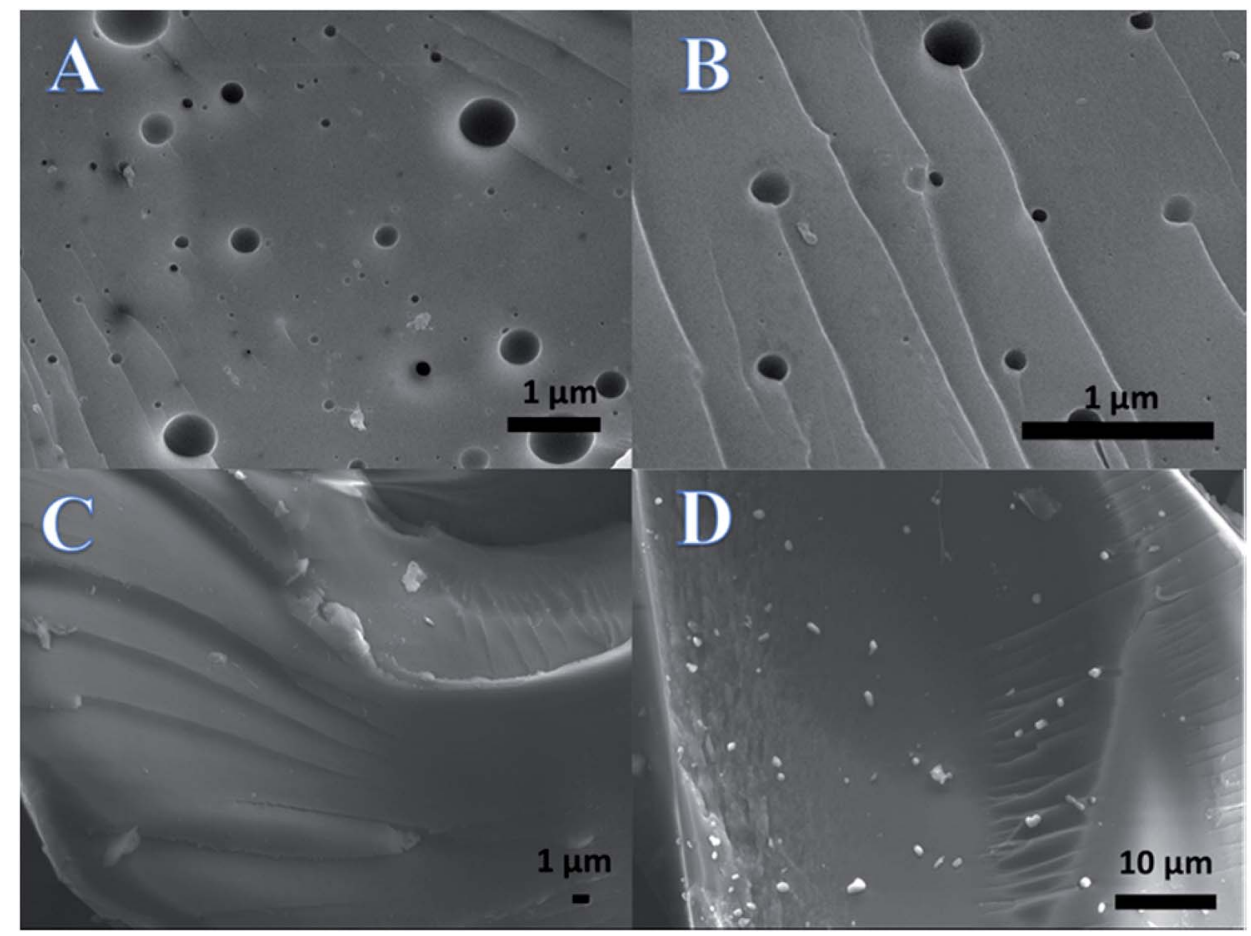

Fig. 3 Scanning electron microscopy images of the hydrogel (A and B) before and after adsorbing (C) Cu ions and (D) Co ions. 


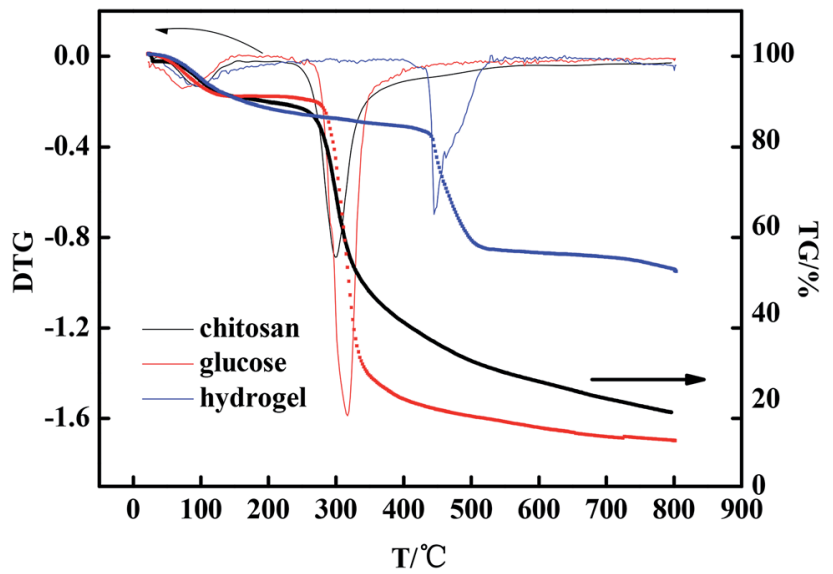

Fig. 4 Thermogravimetric curves of chitosan, glucose and hydrogels

layers of $\mathrm{Cu}$ ions as seen in Fig. 3C and Co ions as seen in Fig. 3D. The disappearance of pores on the hydrogel and the thin metal films indicate that the hydrogel adsorbs $\mathrm{Cu}$ and $\mathrm{Co}$ ions.

\subsection{TGA}

From the thermogravimetric maps of the three substances in Fig. 4, it can be seen that there were two processes. The first one occurred at about $100{ }^{\circ} \mathrm{C}$; this process was the volatilization of water present in the substance, which was full of crystalline water. The second one was the thermal decomposition of the substance at higher temperatures.

The volatilization rate of chitosan reached the maximum at $86{ }^{\circ} \mathrm{C}$ and that of glucose was at $71{ }^{\circ} \mathrm{C}$, and the water loss rate was about $10 \%$. The volatilization rate of water in the hydrogel reached its maximum at $93^{\circ} \mathrm{C}$. The reason for this change is the presence of crystalline water in hydrogels, which contain about 14\% water. The high temperature decomposition of hydrogels was significantly delayed compared with that of chitosan and glucose. The maximum weight loss rate of chitosan occurred at $300{ }^{\circ} \mathrm{C}$ and that of glucose was at $314^{\circ} \mathrm{C}$. The thermal stability of the hydrogel was better than that of the former two. The hydrogel has a high thermal stability. The maximum weight loss rate of the hydrogel was $445{ }^{\circ} \mathrm{C}$, and the final weight of the hydrogel remained at $50 \%$.

\subsection{Adsorption properties of GL/CS hydrogels}

3.5.1 pH. In the application of hydrogels to adsorb heavy metal ions, $\mathrm{pH}$ is an important factor. Studying the adsorption properties of hydrogels at different $\mathrm{pH}$ values can help in the application of hydrogels to treat heavy metal ions in practice. From Fig. 5, it can be seen that the adsorption capacity of the hydrogel increased gradually with the increase in $\mathrm{pH}$ from 1 to 7. At a low $\mathrm{pH}$, the adsorption capacity of hydrogels was relatively small due to the large amount of $\mathrm{H}^{+}$adhering to the surface of hydrogels, and these positive charges repel the heavy metal ions near the hydrogels, resulting in the decrease of the adsorption capacity towards heavy metal ions. With the increase in $\mathrm{pH}$, the concentration of $\mathrm{H}^{+}$and the corresponding rejection

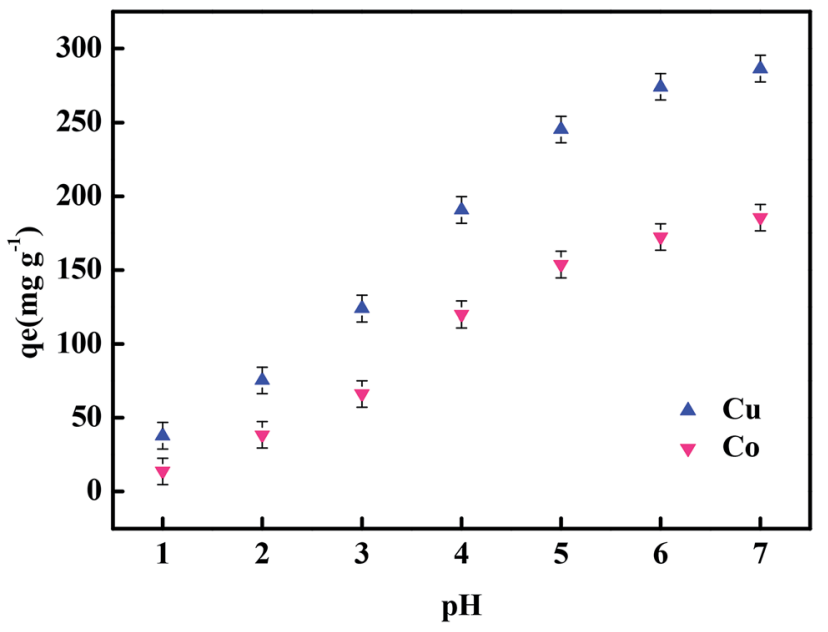

Fig. 5 Effect of $\mathrm{pH}$ on the adsorption properties of the hydrogel.

decrease, and the adsorption capacity increases. At the same time, $\mathrm{H}^{+}$can bind with some hydrogen-containing functional groups, reducing the active sites of adsorption, and thus, the adsorption capacity towards heavy metal ions. ${ }^{48-51}$

3.5.2 Adsorbent dosage. The dose of the adsorbent is an important parameter because it can adsorb only up to a certain capacity at a given initial metal ion concentration. Fig. 6 shows that when the adsorbent dosage is less than $0.02 \mathrm{~g}$, the adsorption capacity towards $\mathrm{Cu}$ (II) and $\mathrm{Co}(\mathrm{II})$ increases with the increase in the adsorbent dosage due to the presence of sufficient adsorption sites on the hydrogel adsorbent. However, when the adsorbent dosage is higher than $0.02 \mathrm{~g}$, the number of sites available on the hydrogels increased with the increase in the adsorbent dosage. Therefore, a number of active sites remained unsaturated, leading to a downward trend. Therefore, the adsorbent dosage of $0.02 \mathrm{~g}$ was chosen as the best.

3.5.3 Study of time effect and dynamic model. The liquidsolid contact time is one of the key parameters for validating that the adsorbent has been successfully applied in wastewater treatment. Fig. 7A shows that the adsorption capacity of

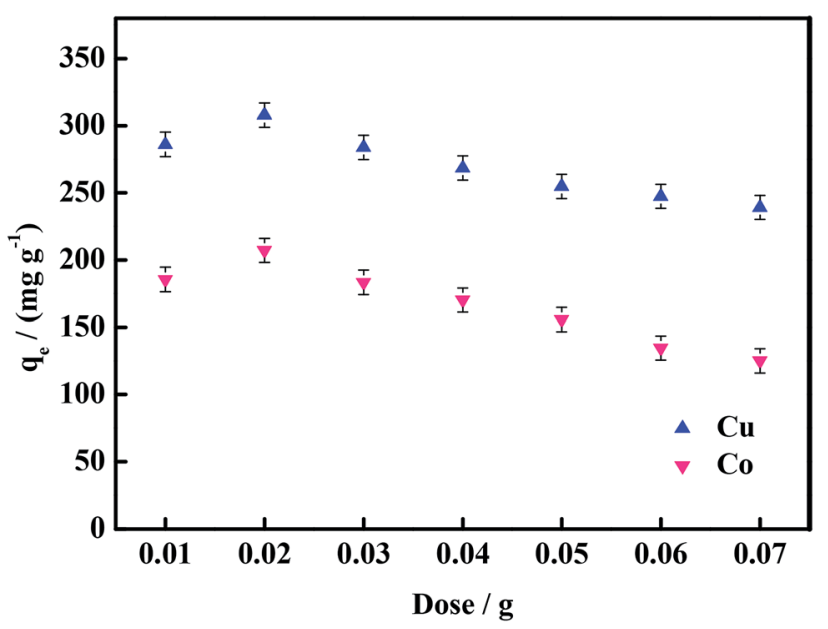

Fig. 6 Effect of adsorbent dosage on the ion adsorption behavior of the hydrogel. 

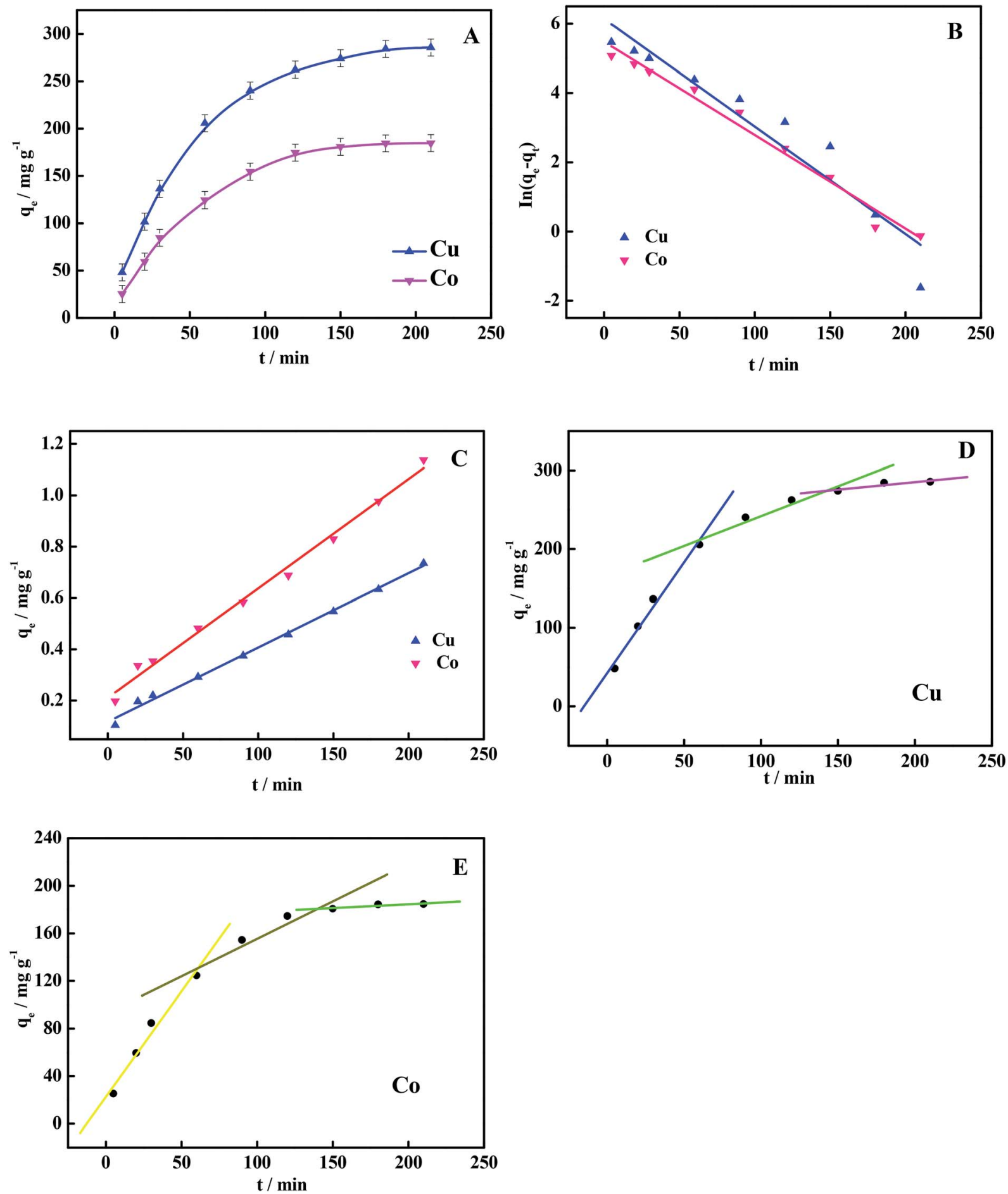

Fig. 7 (A) Effect of time on adsorption, and fitting of (B) pseudo-first-order kinetic model, (C) pseudo-second-order kinetic model and (D and E) intraparticle diffusion models.

hydrogels towards heavy metal ions varies with time and fits the kinetics of the adsorption process. The results showed that the adsorption capacity per unit mass of the adsorbent increased rapidly in $120 \mathrm{~min}$ and slowly reached saturation in $180 \mathrm{~min}$. It consisted of two stages: the initial fast adsorption stage and the final slow adsorption stage. Initially, all the active sites on the adsorbent surface were empty, and the concentration of heavy metal ions was very high. At this time, it was observed that the adsorption capacity increased rapidly. At the slow stage, there were fewer active sites on the adsorbent, so the adsorption capacity increased slowly. For all equilibrium experiments, 180 min contact time was selected.

Adsorption kinetics is an important aspect of the adsorption process, and the kinetic model controls the mechanism of the adsorption process. The pseudo-first-order and pseudo-secondorder kinetic models are discussed below using eqn (2) and (3). 
The equation for the pseudo-first-order kinetic model is as follows:

$$
\operatorname{In}\left(q_{\mathrm{e}}-q_{t}\right)=\operatorname{In} q_{\mathrm{e}}^{*}-k_{1} t
$$

The equation for the pseudo-second-order kinetic model is as follows:

$$
\frac{t}{q_{t}}=\frac{1}{k_{2} q_{\mathrm{e}}^{* 2}}+\frac{t}{q_{\mathrm{e}}^{*}}
$$

where $q_{\mathrm{e}}\left(\mathrm{mg} \mathrm{g}^{-1}\right)$ is the experimentally determined equilibrium adsorption capacity, $q_{\mathrm{e}}^{*}\left(\mathrm{mg} \mathrm{g}^{-1}\right)$ is the theoretical equilibrium adsorption capacity, $t$ ( $\mathrm{min}$ ) is the adsorption time, and $k_{1}$ $\left(\mathrm{min}^{-1}\right)$ and $k_{2}\left(\mathrm{~g} \mathrm{mg}^{-1} \mathrm{~min}^{-1}\right)$ are the rate constants for the first-order and second-order kinetic models, respectively.

$$
q_{t}=k_{\mathrm{p}} t^{1 / 2}+C
$$

where, $q_{t}\left(\mathrm{mg} \mathrm{g}^{-1}\right)$ is the adsorption capacity at time $t, t$ is the time of adsorption, $k_{\mathrm{p}}\left(\mathrm{mg} \mathrm{g}^{-1} \mathrm{~min}^{-1 / 2}\right)$ is the intraparticle diffusion rate constant, and $C$ is a constant related to the boundary layer thickness. The larger the $k_{\mathrm{p}}$ value, more likely is the diffusion of the adsorbate inside the adsorbent. If $C=0$, intraparticle diffusion is the only controlling step; other values indicate that the adsorption process is controlled by multiple steps. Moreover, the larger the $C$, the greater the effect of the boundary layer on adsorption, that is, the greater the effect of membrane diffusion on the adsorption process.

Fitting parameters of the three kinetic models are listed in Table 3. Firstly, for the pseudo-first-order and pseudo-secondorder kinetic models, it can be seen that the pseudo-secondorder kinetic model was more consistent with the actual adsorption process. Its correlation coefficient $\left(R^{2}>0.99\right)$ was

Table 3 Adsorption kinetic parameters

\begin{tabular}{lcc}
$\begin{array}{l}\text { Kinetic models and } \\
\text { parameters }\end{array}$ & $\mathrm{Cu}^{2+}$ & $\mathrm{Co}^{2+}$ \\
\hline$q_{e}\left(\mathrm{mg} \mathrm{g}^{-1}\right)$ & 286.2 & 185.5
\end{tabular}

\section{Pseudo-first-order kinetics}

$q_{\mathrm{e}}^{*}\left(\mathrm{mg} \mathrm{g}^{-1}\right)$

$k_{1}\left(\min ^{-1}\right)$

$R^{2}$

462.2

0.031

0.907

Pseudo-second-order kinetics

$q_{\mathrm{e}}^{*}\left(\mathrm{mg} \mathrm{g}^{-1}\right)$

$k_{2}\left(\mathrm{~g} \mathrm{mg}^{-1} \min ^{-1}\right) \times 10^{5}$

$R^{2}$

Intraparticle diffusion

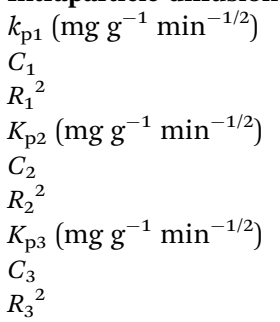

344.5

7.198

0.995

2.818

41.99

0.978

0.758

166.01

0.929

0.191

246.97

0.684
241.1

0.027

0.979

234.7

8.619

0.991

1.775

22.47

0.960

92.39

0.631

0.889

171.65

0.065

0.596 better than that of the pseudo-first-order kinetic model $\left(R^{2}<\right.$ 0.99). It can be seen from the intraparticle diffusion parameters that the adsorption rate was controlled not only by intraparticle diffusion, but also by other steps. ${ }^{52,53}$

3.5.4 Effect of heavy metal ion concentration and study of isotherm model. Fig. 8 shows that at low initial heavy metal ion concentrations, the absorption sites on the adsorbent were easily occupied by $\mathrm{Cu}(\mathrm{II})$ and $\mathrm{Co}(\mathrm{II})$ ions. However, with the increase in the initial concentration of heavy metal ions, most of the available adsorption sites were occupied, resulting in a slow increase of the adsorption capacity. With the increase in the initial concentration of $\mathrm{Cu}$ (II) and $\mathrm{Co}$ (II) ions, the adsorption capacity of hydrogels for the heavy metal ions increased from 286 to $362 \mathrm{mg} \mathrm{g}^{-1}$ and from 184 to $262 \mathrm{mg} \mathrm{g}^{-1}$, respectively. This phenomenon may be related to the increase in the electrostatic interaction at the vacant active sites in the hydrogels.

Isotherm is an important factor for understanding the adsorption efficiency and exploring the adsorption mechanism. The adsorption isotherm explores the relationship between the adsorption capacity and concentration at adsorption equilibrium, that is to say, adsorption capacity is only related to the equilibrium concentration at constant temperature. Following is the discussion on the Langmuir adsorption isotherm model and Freundlich adsorption isotherm model, and understanding the adsorption mechanism.

The linear Langmuir adsorption isotherm equation is as follows:

$$
\frac{C_{\mathrm{e}}}{q_{\mathrm{e}}}=\frac{C_{\mathrm{e}}}{q_{\mathrm{m}, \mathrm{L}}}+\frac{1}{K_{\mathrm{L}} q_{\mathrm{m}, \mathrm{L}}}
$$

where $C_{\mathrm{e}}\left(\mathrm{mg} \mathrm{L}^{-1}\right)$ is the equilibrium concentration, $q_{\mathrm{e}}\left(\mathrm{mg} \mathrm{g}^{-1}\right)$ is the equilibrium adsorption capacity, and $q_{\mathrm{m}, \mathrm{L}}\left(\mathrm{mg} \mathrm{g}^{-1}\right)$ is the maximum saturated adsorption capacity. $K_{\mathrm{L}}\left(\mathrm{L} \mathrm{mg}^{-1}\right)$ is the Langmuir adsorption constant related to the adsorption energy.

The linear Freundlich adsorption isotherm equation is as follows:

$$
\text { In } q_{e}=\operatorname{In} K_{\mathrm{F}}+\frac{1}{n} \operatorname{In} C_{\mathrm{e}}
$$

where $C_{\mathrm{e}}\left(\mathrm{mg} \mathrm{L}^{-1}\right)$ is the equilibrium concentration, $q_{\mathrm{e}}\left(\mathrm{mg} \mathrm{g}^{-1}\right)$ is the equilibrium adsorption capacity, $K_{\mathrm{F}}\left(\mathrm{L} \mathrm{g}^{-1}\right)$ is the Freundlich adsorption constant related to the adsorption capacity, and $n$ is the Freundlich constant related to the degree of surface inhomogeneity.

Temkin adsorption isotherm model is based on the interaction between the adsorbent and the adsorbate. It assumes that the adsorption heat decreases linearly with the increase in the degree of adsorption, and the linear expression is as follows:

$$
q_{\mathrm{e}}=B \operatorname{In} A+B \operatorname{In} C_{\mathrm{e}}
$$

where $C_{\mathrm{e}}\left(\mathrm{mg} \mathrm{L}^{-1}\right)$ is the equilibrium concentration, $q_{\mathrm{e}}\left(\mathrm{mg} \mathrm{g}^{-1}\right)$ is the equilibrium adsorption capacity, $A\left(\mathrm{mg} \mathrm{L}^{-1}\right)$ is the equilibrium constant related to binding energy, and $B$ is the Temkin constant related to the adsorption heat. 

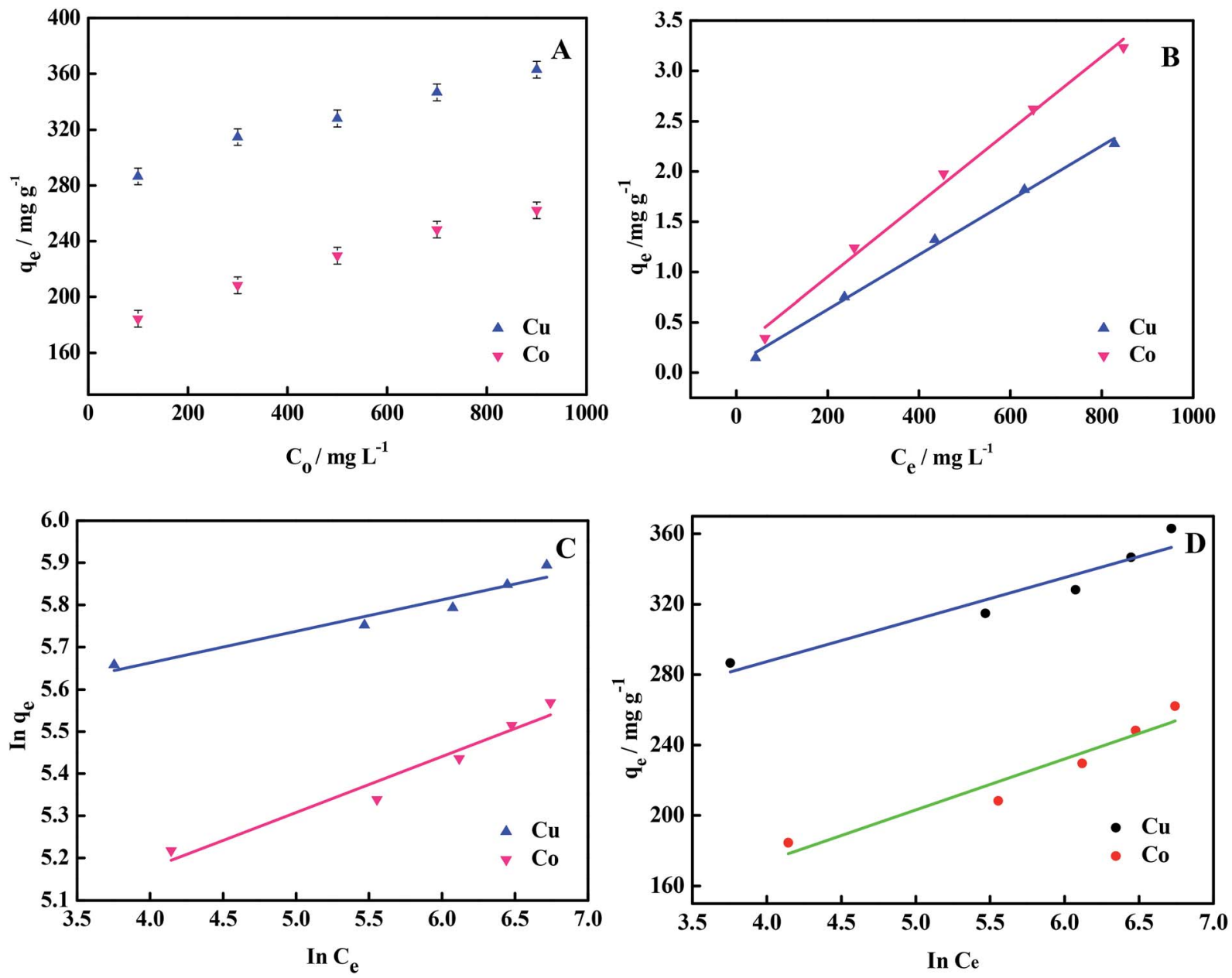

Fig. 8 (A) Effect of heavy metal ion concentration on the adsorption performance, and fitting of (B) Langmuir isotherm, (C) Freundlich isotherm and (D) Temkin isotherm. The experiment was carried out at different initial concentrations of $\mathrm{Cu}(I)$ and $\mathrm{Co}(॥)$ ions ranging from 100 to $900 \mathrm{mg}^{-1}$.

Fitting results for the three isotherm models are listed in Table 4. Different isotherms have different adsorption mechanisms and models. From Table 4, it can be seen that the process by which the hydrogel adsorbs the two metal ions was similar to that of the Langmuir model. From the data point of view, the correlation coefficient for Langmuir model is the largest, and

Table 4 Adsorption isotherm parameters

\begin{tabular}{lcc}
\hline Parameters & $\mathrm{Cu}^{2+}$ & $\mathrm{Co}^{2+}$ \\
\hline Langmuir & & \\
$q_{\mathrm{m}, \mathrm{L}}\left(\mathrm{mg} \mathrm{g}^{-1}\right)$ & 369.1 & 273.9 \\
$K_{\mathrm{L}}\left(\mathrm{L} \mathrm{mg}^{-1}\right)$ & 0.032 & 0.016 \\
$R^{2}$ & 0.995 & 0.991 \\
& & \\
Freundlich & & 103.9 \\
$K_{\mathrm{F}}\left(\mathrm{L} \mathrm{g}^{-1}\right)$ & 213.5 & 0.133 \\
$1 / n$ & 0.075 & 0.937 \\
$R^{2}$ & 0.919 & \\
& & \\
Temkin & & 7.37 \\
$A\left(\mathrm{mg} \mathrm{L}^{-1}\right)$ & 3031 & 29.03 \\
$B$ & 23.91 & 0.907 \\
$R^{2}$ & 0.894 &
\end{tabular}

the experimental data are similar to the model described by Langmuir. From the theoretical view point, the theoretical adsorption capacity calculated using the Langmuir model is the closest to the experimental results. The results of isotherm model fitting are consistent with those obtained above from kinetic modeling studies and FTIR spectroscopy. The adsorption of heavy metal ions by the hydrogel is a chemical adsorption process. ${ }^{19,54}$

3.5.5 Effect of temperature on the adsorption of heavy metal ions by hydrogels and thermodynamic study. As seen from Fig. 9A, the adsorption increases with the increase in the temperature. This may be due to the acceleration of the slow adsorption step or due to the creation of new vacant active sites on the surface of the hydrogel. In order to study the thermodynamics, the obtained data was fitted by the formula in eqn (8). The results are shown in Fig. 9. The corresponding thermodynamic parameters, Gibbs free energy $\Delta G^{\theta}$, entropy change $\Delta S^{\theta}$ and enthalpy change $\Delta H^{\theta}$ are shown in Table 5.

$$
\begin{aligned}
& \Delta G^{\theta}=\Delta H^{\theta}-T \Delta S^{\theta} \\
& \operatorname{In}\left(\frac{q_{\mathrm{e}}}{C_{\mathrm{e}}}\right)=\frac{\Delta S^{\theta}}{R}-\frac{\Delta H^{\theta}}{R T}
\end{aligned}
$$


where $C_{\mathrm{e}}\left(\mathrm{mg} \mathrm{L}^{-1}\right)$ is the equilibrium ion concentration, $q_{\mathrm{e}}(\mathrm{mg}$ $\mathrm{g}^{-1}$ ) is the equilibrium adsorption capacity, $R$ is the universal gas constant, and $T(\mathrm{~K})$ is the thermodynamic temperature.

It can be seen from Table 5 that the $\Delta G^{\theta}$ values were negative and decreased with the increase in temperature, which indicates that the adsorption was a spontaneous process and the increase in temperature was conducive to adsorption, and is consistent with the trend of $q_{\mathrm{e}} v s$. T. The positive values for $\Delta H^{\theta}$ indicate that the adsorption process is endothermic. The fitting of thermodynamic data shows that the adsorption process of heavy metal ions by the hydrogel is a spontaneous endothermic process.

\subsection{Regeneration capacity}

As seen from Fig. 10A, the regeneration efficiencies for $\mathrm{Cu}(\mathrm{II})$ and $\mathrm{Co}(\mathrm{II})$ adsorption were $81 \%$ and $74.8 \%$, respectively, after
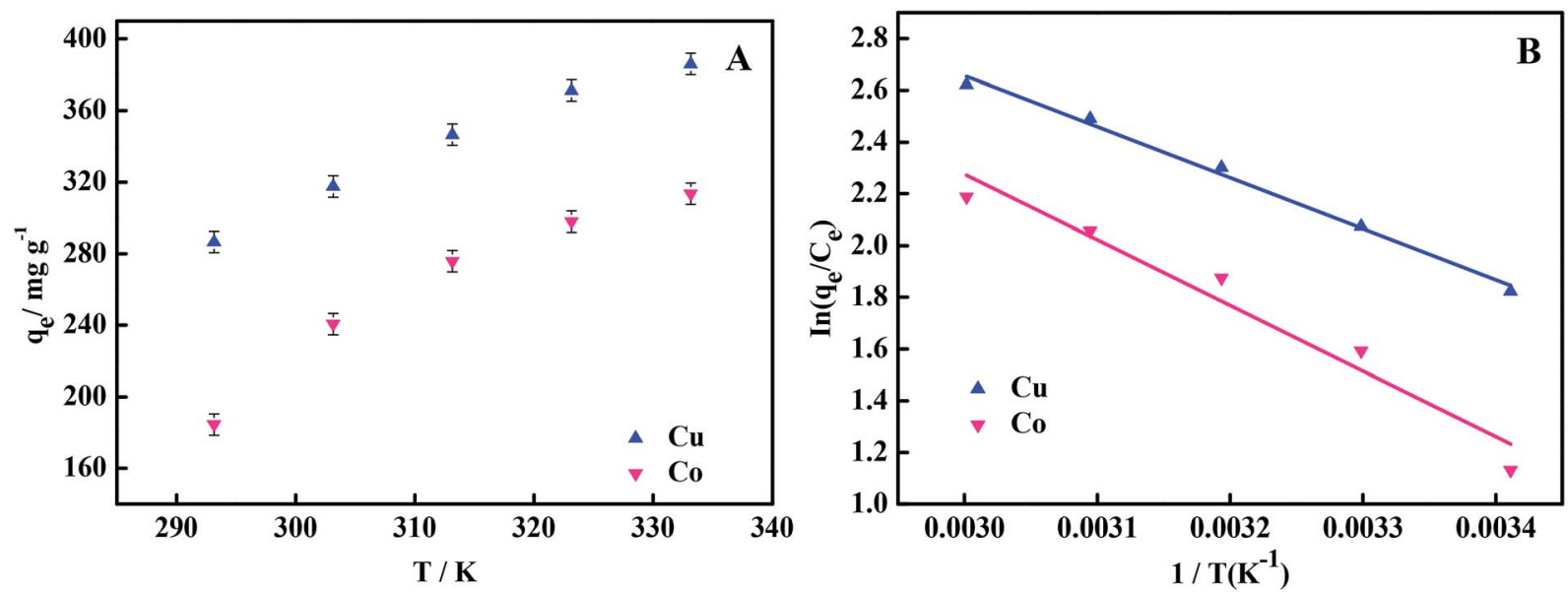

Fig. 9 (A) Effect of temperature of heavy metal ions on adsorption properties of hydrogels and (B) thermodynamics study.

Table 5 Adsorption thermodynamic parameters

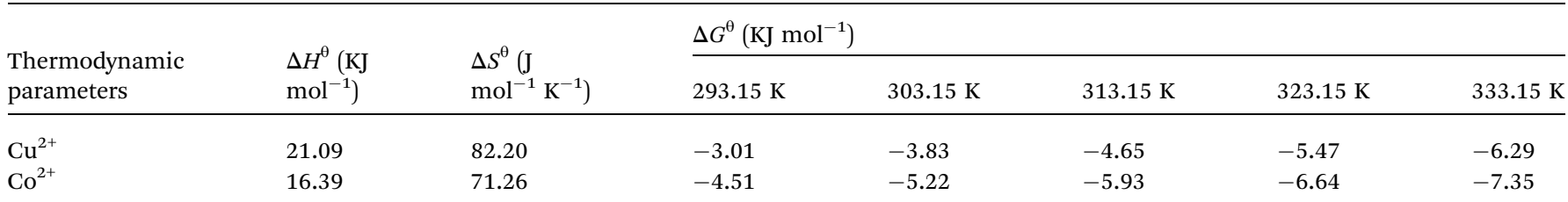
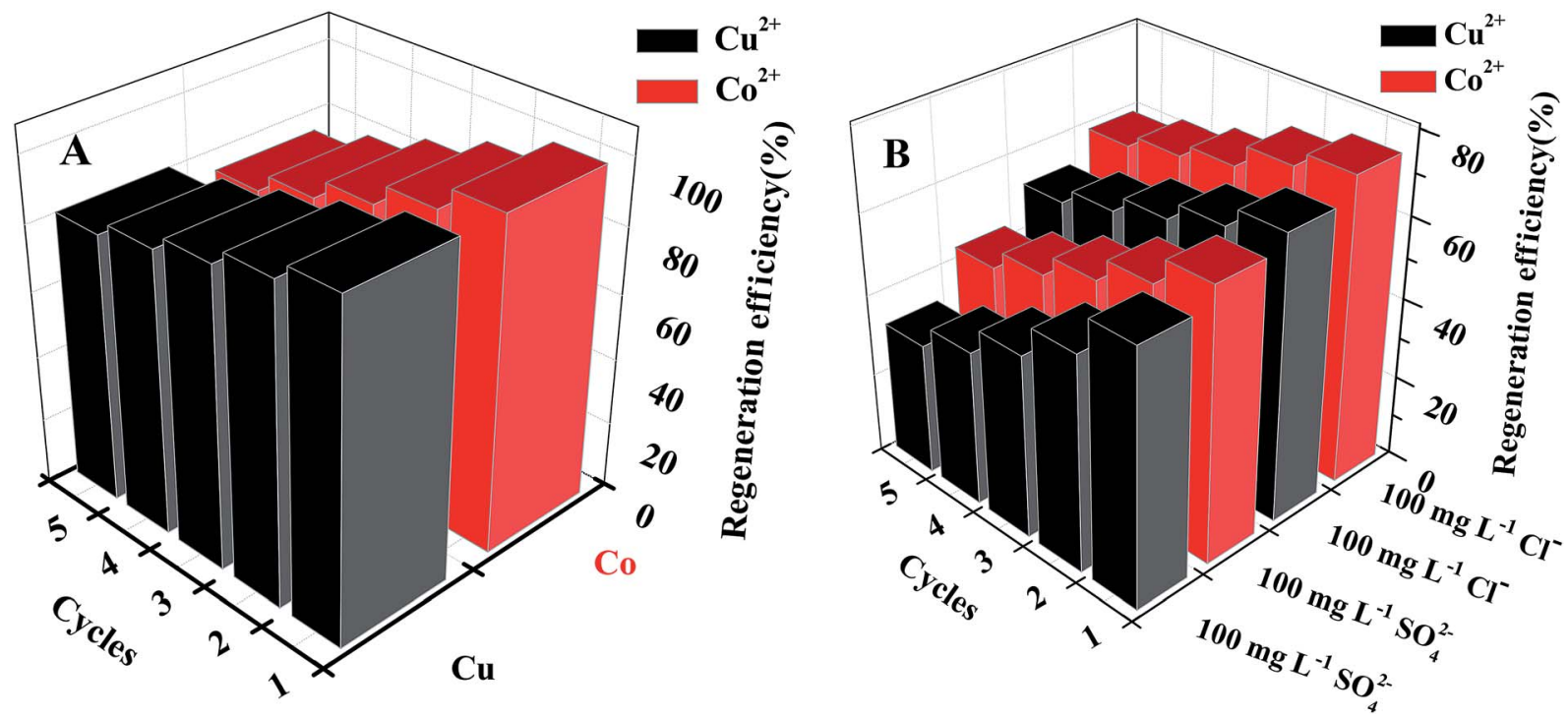

Fig. 10 Hydrogel regeneration efficiency after the adsorption of $\mathrm{Cu}(॥)$ and $\mathrm{Co}(॥)(\mathrm{A})$ and (B) in solutions with different anions. 
Table 6 Comparison of adsorption properties of some adsorbents

\begin{tabular}{lll}
\hline Adsorbent & $q_{\mathrm{e}} / \mathrm{mg} \mathrm{g}^{-1}$ & Reference \\
\hline CMC-hydrogel & $\mathrm{Cu}(\mathrm{II})-142$ & 37 \\
SDS-TT & $\mathrm{Cu}(\mathrm{II})-400$ & 52 \\
IRA-120 & $\mathrm{Co}(\mathrm{II})-166$ & 53 \\
Hydrogels & $\mathrm{Cu}(\mathrm{II})-286$ & This work \\
Hydrogels & $\mathrm{Co}(\mathrm{II})-185$ & This work
\end{tabular}

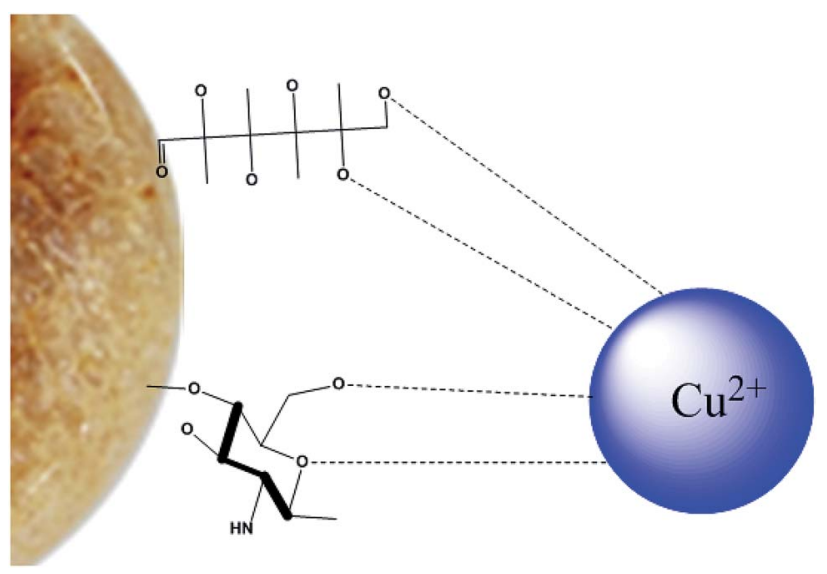

Fig. 11 Adsorption mechanism of heavy metal ions on hydrogel adsorbents.

five cycles, and the loss rate was medium, which means that the hydrogel can be recycled.

When there were other anions in the solution, the cyclic regeneration of hydrogels decreased. The presence of anions obviously affected the adsorption performance of the hydrogel, probably due to the competition for adsorption sites between the anions and heavy metal ions. ${ }^{55}$

Table 6 shows the comparison of adsorption properties of some adsorbents. It can be seen from the table that hydrogel adsorbents have excellent adsorption properties for $\mathrm{Cu}$ and $\mathrm{Co}$. Combined with their recycling ability, the study of hydrogel adsorbents increases the possibility of the application of macromolecule resins for heavy metal ion adsorption.

\subsection{Adsorption mechanism}

The mechanism of adsorption of heavy metal ions by hydrogel composites is shown in Fig. 11. Hydrogel composites have many hydroxyl, amino and carboxyl groups, which make the surface of the hydrogel negatively charged. ${ }^{56}$ Due to electrostatic attraction, the hydrogels and heavy metal ions attract each other, and the copper ions coordinate with these groups.

\section{Conclusions}

In this study, we successfully synthesized a high performance hydrogel with porous structure and excellent adsorption performance using chitosan and glucose for the treatment of heavy metal ions $\mathrm{Cu}$ (II) and $\mathrm{Co}$ (II) in wastewater. The optimal hydrogel synthesis conditions from the orthogonal optimization experiment was $A(70), B(0.2), C(0.05)$, and $D$ (0.03). The influence of the four factors followed the order of $D>$ $B>C>A$. The role of initiator was the most important. The TGA results showed that the hydrogel had high thermal stability. The maximum weight loss rate of the hydrogel was at $445{ }^{\circ} \mathrm{C}$ and the final weight of hydrogel remained at $50 \%$. Based on the adsorption experiments, the adsorption performance was optimal under neutral conditions, and $0.02 \mathrm{~g}$ of adsorbent was chosen as the best dosage. The process of adsorbing $\mathrm{Cu}$ (II) and Co(II) ions follows a pseudo second-order kinetic model and a Langmuir isotherm model, which indicates a spontaneous endothermic reaction. In addition, the hydrogel adsorbent could be recycled, and the regeneration efficiency after five cycles was over $70 \%$.

\section{Conflicts of interest}

There are no conflicts to declare.

\section{Acknowledgements}

We gratefully acknowledge the Science Foundation of North University of China (No. XJJ2016012) for financial support of this research.

\section{References}

1 M. Al-Shannag, Z. Al-Qodah, K. Bani-Melhem, M. R. Qtaishat and M. Alkasrawi, Chem. Eng. J., 2015, 260, 749-756.

2 A. Chan, H. Salsali and E. McBean, ACS Sustainable Chem. Eng., 2013, 2, 130-137.

3 J. Lu, Y. Li, S. Zhang and Y. Sun, J. Hazard. Mater., 2015, 286, 466-473.

4 A. Shah, S. Shahzad, A. Munir, M. N. Nadagouda, G. S. Khan, D. F. Shams, D. D. Dionysiou and U. A. Rana, Chem. Rev., 2016, 116, 6042-6074.

5 L. C. Shen, X. T. Nguyen and N. P. Hankins, Sep. Purif. Technol., 2015, 152, 101-107.

6 F. Fu and Q. Wang, J. Environ. Manage., 2011, 92, 407-418.

7 S. H. Kazemi, B. Karimi, S. A. Aghdam, H. Behzadnia and M. A. Kiani, RSC Adv., 2015, 5, 69032-69041.

8 K. Y. Foo and B. H. Hameed, Chem. Eng. J., 2010, 156, 2-10. 9 J. Shin, Y. Kim, Y. M. Lim and Y. C. Nho, J. Appl. Polym. Sci., 2008, 107, 3179-3183.

10 T. Sakai, T. Matsunaga, Y. Yamamoto, C. Ito, R. Yoshida, S. Suzuki, N. Sasaki, M. Shibayama and U. I. Chung, Macromolecules, 2008, 41, 5379-5384.

11 X. Yi, Z. Xu, Y. Liu, X. Guo, M. Qu and X. Xu, RSC Adv., 2017, 7, 6278-6287.

12 L. Passauer, M. Struch, S. Schuldt, J. Appelt, Y. Schneider, D. Jaros and H. Rohm, ACS Appl. Mater. Interfaces, 2012, 4, 5852.

13 M. Z. Jing, Y. Fu, X. Fei, J. Tian, H. Zhi, H. Y. Zhang, L. Q. Xu, X. Y. Wang and Y. Wang, Polym. Chem., 2017, 8, 3553-3559.

14 M. Tan, T. Zhao, H. Huang and M. Guo, Polym. Chem., 2013, 4, 5570 . 
15 A. J. M. Valente and O. Soderman, Adv. Colloid Interface Sci., 2014, 205, 156-176.

16 Y. Zang, Q. Yue, Y. Kan, L. Zhang and B. Gao, Ecotoxicol. Environ. Saf., 2018, 161, 467-473.

17 T. Garrison, J. C. Hower, A. E. Fryar and E. D'Angelo, Environ. Earth Sci., 2016, 75, 1-13.

18 M. A. Badawi, N. A. Negm, M. T. Abou Kana, H. H. Hefni and A. M. M. Moneem, Int. J. Biol. Macromol., 2017, 99, 465-476.

19 Z. A. AL-Othman, R. Ali and M. Naushad, Chem. Eng. J., 2012, 184, 238-247.

20 M. Naushad, Chem. Eng. J., 2014, 235, 100-108.

21 R. Karthik and S. Meenakshi, Chem. Eng. J., 2015, 263, 168177.

22 R. K. Misra, S. K. Jain and P. K. Khatri, J. Hazard. Mater., 2011, 185, 1508-1512.

23 G. Zhou, C. Liu, L. Chu, Y. Tang and S. Luo, Bioresour. Technol., 2016, 219, 451-457.

24 G. Zhou, J. Luo, C. Liu, L. Chu, J. Ma and Y. Tang, Water Res., 2016, 89, 151-160.

25 A. J. M. Karawi, Z. H. J. Qaisi, H. I. Abdullah, A. M. A. Mokaram and D. T. A. Heetimi, Carbohydr. Polym., 2011, 83, 495-500.

26 A. L. Bolden, C. F. Kwiatkowski and T. Colborn, Environ. Sci. Technol., 2015, 49, 5261-5276.

27 G. A. Płaza, J. Wypych, C. Berry and R. L. Brigmon, World J. Microbiol. Biotechnol., 2007, 23, 533-542.

28 A. V Reis, M. R. Guilherme, A. T. Paulino, E. C. Muniz, L. H. C. Mattoso and E. B. Tambourgi, Langmuir, 2009, 25, 2473-2478.

29 L. P. Ramteke and P. R. Gogate, J. Chem. Technol. Biotechnol., 2016, 91, 456-466.

30 W. Shen, J. Luan, L. Cao, J. Sun, L. Yu and J. Ding, Biomacromolecules, 2015, 16, 105-115.

31 J. Liang, G. Shan and P. Pan, Soft Matter, 2017, 13, 41484158.

32 C. Abasi, A. Abia and J. Igwe, Environ. Res. J., 2011, 5, 104113.

33 V. C. Sanchez, A. Jachak, R. H. Hurt and A. B. Kane, Chem. Res. Toxicol., 2011, 25, 15-34.

34 S. Wang, X. Li, Y. Liu, C. Zhang, X. Tan, G. Zeng, B. Song and L. Jiang, J. Hazard. Mater., 2018, 342, 177-191.

35 Z. Dong, D. Wang, X. Liu, X. Pei, L. Chen and J. Jin, J. Mater. Chem. A, 2014, 2, 5034-5040.

36 E. Guibal, Prog. Polym. Sci., 2005, 30, 71-109.

37 A. Li and A. Wang, Eur. Polym. J., 2005, 41, 1630-1637.
38 K. Sharma, B. Kaith, V. Kumar, S. Kalia, V. Kumar and H. Swart, Geoderma, 2014, 232, 45-55.

39 S. Chatterjee, M. W. Lee and S. H. Woo, Bioresour. Technol., 2010, 101, 1800-1806.

40 C. Gan, Y. Liu, X. Tan, S. Wang, G. Zeng, B. Zheng, T. Li, Z. Jiang and W. Liu, RSC Adv., 2015, 5, 35107-35115.

41 A. Rawal, S. D. Joseph, J. M. Hook, C. H. Chia, P. R. Munroe, S. Donne, Y. Lin, D. Phelan, D. R. G. Mitchell, B. Pace, J. Horvat and J. B. W. Webber, Environ. Sci. Technol., 2016, 50, 7706-7714.

42 J. Deng, B. Lei, A. He, X. Zhang, L. Ma, S. Li and C. Zhao, J. Hazard. Mater., 2013, 263, 467-478.

43 W. Cui, J. Ji, Y.-F. Cai, H. Li and R. Ran, J. Mater. Chem. A, 2015, 3, 17445-17458.

44 C. M. C. Filho, M. N. L. Neto, R. S. Teixeira, A. A. C. C. Pais and A. J. M. Valente, J. Liq. Chromatogr. Relat. Technol., 2016, 39, 837-846.

45 M. Sulak, E. Demirbas and M. Kobya, Bioresour. Technol., 2007, 98, 2590-2598.

46 W. S. Wan Ngah, L. C. Teong and M. A. K. M. Hanafiah, Carbohydr. Polym., 2011, 83, 1446-1456.

47 H. Sereshti, S. Samadi, S. Asgari and M. Karimi, RSC Adv., 2015, 5, 9396-9404.

48 G. Mezohegyi, F. P. van der Zee, J. Font, A. Fortuny and A. Fabregat, J. Environ. Manage., 2012, 102, 148-164.

49 M. A. Kamal, S. Bibi, S. W. Bokhari, A. H. Siddique and T. Yasin, React. Funct. Polym., 2017, 110, 21-29.

50 Q. Du, J. Sun, Y. Li, X. Yang, X. Wang, Z. Wang and L. Xia, Chem. Eng. J., 2014, 245, 99-106.

51 Y. Yao, S. Miao, S. Liu, L. P. Ma, H. Sun and S. Wang, Chem. Eng. J., 2012, 184, 326-332.

52 M. Naushad, Z. A. ALOthman, M. M. Alam, M. R. Awual, G. E. Eldesoky and M. Islam, J. Iran. Chem. Soc., 2015, 12, 1677-1686.

53 M. Naushad, Z. A. ALOthman, G. Sharma and Inamuddin, Ionics, 2015, 21, 1453-1459.

54 A. A. Alqadami, M. Naushad, M. A. Abdalla, T. Ahamad, Z. A. ALOthman, S. M. Alshehri and A. A. Ghfar, J. Cleaner Prod., 2017, 156, 426-436.

55 H. Liu, F. Zhang and Z. Y. Peng, Sci. Rep., 2019, 9, 3663-3675. 56 M. Naushad, T. Ahamad, G. Sharma, A. H. Al-Muhtaseb, A. B. Albadarin, M. M. Alam, Z. A. ALOthman, S. M. Alshehri and A. A. Ghfar, Chem. Eng. J., 2016, 300, 306-316. 